DEVELOPMENTS IN BESSEL'S FUNCTIONS. [Oct.,

\title{
ON THE DEVELOPMENTS IN BESSEL'S FUNCTIONS.
}

BY PROFESSOR CHARLES N. MOORE.

(Read before the American Mathematical Society, January 1, 1916.)

IN studying the developments in Bessel's functions it is found that the behavior of the series at the origin and in a neighborhood including the origin is, in certain cases, difficult to determine. For example most of the proofs of the convergence of these developments leave unsettled the question of the convergence at the origin of the developments in Bessel's functions of order zero. Also, most of the discussions of the uniform convergence of these developments leave unsettled the question of the uniform convergence of the developments in a neighborhood including the origin. Yet for many of the applications to mathematical physics it is essential to settle both of these points in order to be sure that we have a solution of the physical problem we are discussing.

It was a consideration of these facts that led the writer several years ago to devise a method of establishing the convergence of the developments in Bessel's functions that would settle both of these questions under conditions sufficiently wide for the applications.* The method referred to, however, serves only to establish the convergence or uniform convergence of the developments, and it is necessary to determine the value to which they converge by means of other considerations. In the paper just referred to this was done by using the results of previous writers in connection with the value of the developments. As these results are obtained by means of long and complicated discussions, it seems highly desirable to have some fairly simple method of determining the value of the development when we know it converges. Such a method is given in the present paper, and consequently this paper combined with the previous one gives the first complete discussion of the convergence and value of the developments in Bessel's functions under conditions that are usually satisfied in the applications.

* Cf. Transactions, vol. 12 (1911), p. 181. 
The method used here was first suggested by Sturm and Liouville in connection with the Sturm-Liouville expansions, and the application of it to these expansions was sketched out by them.* Some forty years later Heine attempted to apply this method to the developments in Bessel's functions. $\dagger$ His treatment of this case is inadequate, however, for by omitting practically all the details he avoids all the difficulties. Moreover, it is apparent that he did not himself examine the details of the proof with sufficient care, for he fails to note the presence of an extra term in the development in certain exceptional cases, a fact first observed by Dini.ł

The cases just mentioned are not essentially exceptional but only appear so on account of the notation ordinarily employed. As pointed out before, $\S$ they can be included in the general treatment by means of adopting the following notation. We set

$$
F_{\nu}(\lambda, x) \equiv\left(\frac{x}{2}\right)^{\nu} \sum_{i=0}^{\infty} \frac{(-1)^{i}(\lambda x)^{2 i}}{2^{2 i} i ! \Gamma(\nu+i+1)} \equiv \frac{1}{\lambda^{\nu}} J_{\nu}(\lambda x)
$$

and consider the series

$$
\sum_{n=1}^{\infty} F_{\nu}\left(\lambda_{n}, x\right) \frac{\int_{0}^{1} x f(x) F_{\nu}\left(\lambda_{n}, x\right) d x}{\int_{0}^{1} x\left[F_{\nu}\left(\lambda_{n}, x\right)\right]^{2} d x}=\sum_{n=1}^{\infty} A_{n} F_{\nu}\left(\lambda_{n}, x\right),
$$

where the $\lambda$ 's are the successive roots, positive or zero of the equation

$$
\omega(\lambda)=\left[l \frac{\partial}{\partial x} F_{\nu}(\lambda, x)+h F_{\nu}(\lambda, x)\right]_{x=1}=0,
$$

arranged in increasing order of magnitude.

The object of the present paper is to show that whenever the series (2) converges or is summable at all points of the interval $0 \leqq x \leqq 1$ at which $f(x)$ is continuous, then under certain conditions to be stated subsequently the value of the series at each of these points will be $f(x)$. We will begin by establishing some lemmas.

* Cf. Liouville's Journal, vol. 2 (1837), p. 220.

† Cf. Crelle's Journal, vol. 89 (1880), p. 35.

$\ddagger$ Dini, Serie di Fourier, Pisa, 1880.

§ Cf. Transactions, vol. 10 (1909), p. 420, and vol. 12 (1911), p. 182. 
LEMma 1. The integrals

$$
\begin{gathered}
\frac{1}{2 \pi i} \int_{R_{n}} \frac{z F_{\nu}(z, x)}{(z-\alpha) \omega(z)} d z, \\
\frac{1}{2 \pi i} \int_{R_{n^{\prime}}} \frac{F_{\nu}(z, x)-x^{\nu} F_{\nu}(z, 1)}{(z-\alpha) z^{2} F_{\nu}(z, 1)} d z \quad \text { ( } \alpha \text { a real constant), }
\end{gathered}
$$

where $\omega(z)$ is defined by (3), l not being zero, and the path of integration is a rectangle in the complex plane with vertices at the points $(k, \sqrt{k}),(-k, \sqrt{k}),(-k,-\sqrt{k})$ and $(k,-\sqrt{k})$, $k$ being chosen equal to

(5) $n \pi+\frac{2 \nu+3}{4} \pi$ or $n \pi+\frac{2 \nu+1}{4} \pi$ (n a positive integer),

according as we are considering the first integral or the second integral, will approach zero as $n$ becomes infinite, provided $x$ lies in the interval $0<x<1$.

We shall give the proof in detail for the first only of the integrals in (4), the treatment of the second integral being analogous.

We have for $J_{\nu}(z)$ the following asymptotic expansion*

(6) $J_{\nu}(z)=\frac{1}{\sqrt{2 \pi z}}\left[e^{i(z-\gamma)}\left(1+\theta_{1}(z, \nu)\right)+e^{-i(z-\gamma)}\left(1+\theta_{2}(z, \nu)\right)\right]$, where $\gamma=\frac{1}{4}(2 \nu+1) \pi$ and

$$
\left|\theta_{1}(z, \nu)\right|<\frac{K_{1}}{|z|}, \quad\left|\theta_{2}(z, \nu)\right|<\frac{K_{2}}{|z|} \quad\left(\begin{array}{c}
|z|>c>0 \\
R(z)>0
\end{array}\right)
$$

$K_{1}$ and $K_{2}$ being positive constants for a fixed value of $\nu$.

Making use of (1) and the well-known relation

$$
J_{\nu}^{\prime}(z)=-J_{\nu+1}(z)+\frac{\nu}{z} J_{\nu}(z)
$$

we obtain

* Cf. H. Weber, "Zur Theorie der Bessel'schen Functionen," Math. Annalen, vol. 37 (1890), p. 404 . Equation (6) above results from a combination of equations (19), (30) and (33) of the paper referred to, and is established there for all values of $\nu>-\frac{1}{2}$, and for all values of $z$ whose real part is positive. It can be at once extended to values of $z$ along the axis of imaginaries, the origin being excepted, on account of the continuity of all the functions involved, and for values of $z$ to the left of the axis of imaginaries we can get a corresponding expression by means of the relationship $J_{\nu}(-z)$ $=(-1) \nu J_{\nu}(z)$. 


$$
\omega(z)=\frac{1}{z^{\nu}}\left[(l \nu+h) J_{\nu}(z)-l z J_{\nu+1}(z)\right] .
$$

For the sake of brevity we set

$$
\begin{gathered}
\theta_{1}=\theta_{1}( \pm z, \nu), \quad \theta_{1}^{\prime}=\theta_{1}( \pm z x, \nu), \quad \theta_{2}=\theta_{2}( \pm z, \nu), \\
\theta_{2}^{\prime}=\theta_{2}( \pm z x, \nu), \quad \theta_{3}=\theta_{1}( \pm z, \nu+1), \quad \theta_{4}=\theta_{2}( \pm z, \nu+1) .
\end{gathered}
$$

Then if we further set $z=p+q i$ and make use of (1), (6), the extension of (6) indicated in the previous footnote and (9), we see that the integrand of the first integral in (4) may be written in the form

$$
\begin{aligned}
& \frac{z\left[e^{i( \pm p x-\gamma)} e^{\mp q x}\left(1+\theta_{1}{ }^{\prime}\right)+e^{-i( \pm p x-\gamma)} e^{ \pm q x}\left(1+\theta_{2}{ }^{\prime}\right)\right]}{\sqrt{x}(z-\alpha)\left[\mp l z \left\{e^{i[ \pm p-\gamma-(\pi / 2)]} e^{\mp q}\left(1+\theta_{3}\right)\right.\right.} \\
& \left.+e^{-i[ \pm p-\gamma-(\pi / 2)]} e^{ \pm q}\left(1+\theta_{4}\right)\right\}+(l \nu+h) \\
& \left.\quad \times\left\{e^{i( \pm p-\gamma)} e^{\mp q}\left(1+\theta_{1}\right)+e^{-i( \pm p-\gamma)} e^{ \pm q}\left(1+\theta_{2}\right)\right\}\right]
\end{aligned}
$$

For points on the sides of the rectangle $R_{n}$ that are parallel to the $y$-axis, i. e., for values of $z$ of the form $\pm k+i q$, where $k$ has the first of the values (5), (10) reduces to

$$
(-1)^{n} \frac{e^{\mp q x} e^{i(k x-\gamma)}\left(1+\theta_{1}{ }^{\prime}\right)+e^{ \pm q x} e^{-i(k x-\gamma)}\left(1+\theta_{2}{ }^{\prime}\right)}{\mp l \sqrt{x}\left\{ \pm\left(\left(n+\frac{1}{2}\right) \pi+\gamma\right)-\alpha+i q\right\}\left(e^{q}+e^{-q}\right)\left(1+\theta_{5}\right)},
$$

where for large values of $|z|, \theta_{5}$ is an infinitesimal of the same order as the other $\theta$ 's. It is readily seen from this latter form that if $n$ is chosen large enough to make $\theta_{1}{ }^{\prime}, \theta_{2}{ }^{\prime}$ and $\theta_{5}$ each less in absolute value than $\frac{1}{2}$, the integrand in (4) will for the values of $z$ under consideration be less in absolute value than

$$
\frac{6}{l \sqrt{x}\left\{\left(n+\frac{1}{2}\right) \pi+\gamma-\alpha\right\}} \text {. }
$$

Hence the contributions to the first integral in (4) from the integrations along the sides of the rectangle parallel to the $y$-axis, will be less in absolute value than

$$
\frac{1}{2 \pi} \cdot \frac{24 \sqrt{\left(n+\frac{1}{2}\right) \pi+\gamma}}{l \sqrt{x}\left\{\left(n+\frac{1}{2}\right) \pi+\gamma-\alpha\right\}},
$$

and therefore will approach zero as $n$ becomes infinite. 
For points on the sides of the rectangle $R_{n}$ that are parallel to the $x$-axis, i. e., for values of $z$ of the form $p \pm i \sqrt{k}, k$ having the same value as above, (10) reduces to*

$\frac{e^{i( \pm p x-\gamma)} e^{\mp( \pm) \sqrt{k} x}\left(1+\theta_{1}{ }^{\prime}+e^{-i( \pm p x-\gamma)} e^{ \pm( \pm) \sqrt{k} x}\left(1+\theta_{2}{ }^{\prime}\right)\right.}{\mp l \sqrt{x}\left(p-\alpha+( \pm) i \sqrt{\left(n+\frac{1}{2}\right) \pi+\gamma}\right) e^{\sqrt{k}} e^{\mp( \pm)[[ \pm p-\gamma-(\pi / 2)]}\left(1+\theta_{6}\right)}$,

where for large values of $|z|, \theta_{6}$ is an infinitesimal of the same order as the other $\theta$ 's. It is readily seen from this form that if $n$ is chosen large enough to make $\theta_{1}, \theta_{2}$, and $\theta_{6}$ each less in absolute value than $\frac{1}{2}$, the integrand of the first integral in (4) will for the values of $z$ under consideration be less in absolute value than $\dagger$

$$
\frac{6}{l \sqrt{x\left(\left(n+\frac{1}{2}\right) \pi+\gamma\right)} e^{(1-x) \sqrt{\left(n+\frac{1}{2}\right) \pi+\gamma}}} .
$$

Hence the contributions to the first integral in (4) from the integrations along the sides of the rectangle parallel to the $x$-axis, will be less in absolute value than

$$
\frac{1}{2 \pi} \cdot \frac{24\left\{\left(n+\frac{1}{2}\right) \pi+\gamma\right\}}{l \sqrt{x\left(\left(n+\frac{1}{2}\right) \pi+\gamma\right)} e^{(1-x) \sqrt{\left(n+\frac{1}{2}\right) \pi+\gamma}}},
$$

and therefore will approach zero as $n$ becomes infinite.

Combining the previous results it is seen that the first integral in (4) is for large enough values of $n$ less in absolute value than the sum of (11) and (12). Hence it approaches zero as $n$ becomes infinite, and thus our lemma is proved for this case. As stated before, the proof for the other case is analogous.

LEMMA 2. The function $J_{\nu}(\alpha x)$, where $\alpha$ is a real constant $\neq 0$, can be expanded into a series of the form

$$
\sum_{n=1}^{\infty} B_{n} F_{\nu}\left(\lambda_{n}, x\right),
$$

* The ambiguous signs enclosed in parentheses form an independent set from those not so enclosed.

$\dagger$ For the corresponding values of $z$ the integrand of the second integral in (4) can be shown to be less in absolute value than

$$
\frac{12}{\sqrt{x(n \pi+\gamma)^{3}}} \text {. }
$$


where $\lambda_{1}, \lambda_{2}, \lambda_{3}, \cdots$ are the roots, positive or zero, of equation (3) for the case $l \neq 0$, arranged in increasing order of magnitude, the $B$ 's are constants, and the series converges uniformly in the interval $0 \leqq x \leqq 1$.

Let us set

$$
\frac{\omega(z)}{z}=u(z)=\frac{v(z)}{z^{\nu+1}} .
$$

Then if we make use of Cauchy's theorem of residues, we obtain for the first integral in (4)

$$
\frac{1}{2 \pi i} \int_{R_{n}} \frac{F_{\nu}(z, x)}{(z-\alpha) u(z)} d z=\frac{F_{\nu}(\alpha, x)}{u(\alpha)}+\sum_{\lambda} \frac{F_{\nu}(\lambda, x)}{(\lambda-\alpha) u^{\prime}(\lambda)}
$$

where the summation on the right-hand side is extended over all values of $\lambda$ that are roots of the first order of $u(\lambda)$ and lie within the rectangle $R_{n}$.

Since for every positive root of (3) there is a corresponding negative root, equal in numerical value, there will be two terms on the right-hand side of (15) corresponding to each positive root of (3). We shall now combine these two terms into one of the same type as the general term of (13).

Since from (14) any positive root of $\omega(z)$ is also a positive root of $u(z)$ and $v(z)$, we have for any positive root $\lambda$ of (3)

$$
u^{\prime}(\lambda)=\frac{v^{\prime}(\lambda)}{\lambda^{\nu+1}}-\frac{(\nu+1) v(\lambda)}{\lambda^{\nu+2}}=\frac{v^{\prime}(\lambda)}{\lambda^{\nu+1}} .
$$

But from (14) and (9)

$$
v^{\prime}(\lambda)=(l \nu+h) J_{\nu}{ }^{\prime}(\lambda)-l J_{\nu+1}(\lambda)-l \lambda J^{\prime}{ }_{\nu+1}(\lambda),
$$

which by means of (8) and another well-known formula,

$$
J^{\prime}{ }_{\nu+1}(z)=J_{\nu}(z)-\frac{\nu+1}{z} J_{\nu+1}(z)
$$

may be put in the form

$$
v^{\prime}(\lambda)=-h J_{\nu+1}(\lambda)+\frac{l\left(\nu^{2}-\lambda^{2}\right)+h \nu}{\lambda} J_{\nu}(\lambda) .
$$

Moreover, since $\lambda$ is a positive root of (3) and the $l$ of (3) has been assumed to be not zero, it follows from (9) that

$$
J_{\nu+1}(\lambda)=\frac{l \nu+h}{\lambda \lambda} J_{\nu}(\lambda) \text {. }
$$


Combining (16), (17) and (18), we obtain

$$
u^{\prime}(\lambda)=\frac{l^{2}\left(\nu^{2}-\lambda^{2}\right)-h^{2}}{\lambda^{\nu+2}} J_{\nu}(\lambda) .
$$

It follows from (1) and (19) that

$$
F_{\nu}(-\lambda, x)=F_{\nu}(\lambda, x), \quad u^{\prime}(-\lambda)=u^{\prime}(\lambda) .
$$

Hence the two terms on the right-hand side of (15) corresponding to $\lambda$ and $-\lambda$ combine into the form

$$
\frac{2 \alpha \lambda \lambda^{2}}{\left(\lambda^{2}-\alpha^{2}\right)\left\{l^{2}\left(\nu^{2}-\lambda^{2}\right)-h^{2}\right\} F_{\nu}(\lambda, 1)} F_{\nu}(\lambda, x) .
$$

We know from Lemma 1 that for values of $x$ in the interval $0<x<1$ the left-hand side of equation (15) approaches zero as $n$ becomes infinite. Hence the right-hand side does also, and if we take limits we obtain for $J_{\nu}(\alpha x)$ a series of the form (13),* where

$$
B_{n}=\frac{2 \alpha^{\nu+1} u(\alpha) l \lambda^{2}}{\left(\lambda^{2}-\alpha^{2}\right)\left[l^{2}\left(\lambda^{2}-\nu^{2}\right)+h^{2}\right] F_{\nu}(\lambda, 1)},
$$

and we know that the series converges to $J_{\nu}(\alpha x)$ in the interval $0<x<1$. It follows at once from the form of $B_{n}$ and wellknown properties of the roots of (3) that the series converges uniformly throughout the interval $0 \leqq x \leqq 1$, and hence it represents $J_{\nu}(\alpha x)$ throughout that interval. Our lemma is therefore proved.

Lemma 3. The function $J_{\nu}(\alpha x)-x^{\nu} J_{\nu}(\alpha)$, where $\alpha$ is a real constant $\neq 0$, can be expanded into a series of the form (13) where $\lambda_{1}, \lambda_{2}, \lambda_{3}, \cdots$ are the successive positive roots of equation (3) for the case $l=0$, arranged in increasing order of magnitude, the B's are constants, and the series converges uniformly in the interval $0 \leqq x \leqq 1$.

If we treat the second integral in (4) in the same way as we treated the first integral in the proof of Lemma 2, we obtain for the function $J_{\nu}(\alpha x)-x^{\nu} J_{\nu}(\alpha)$ a series of the form (13) where

* It is apparent from (9) and (14) that $u(z)$ will have a zero root of the first order when and only when (3) has a zero root. As would be expected, the coefficient of the general term of the series (2) for the case where $f(x)=J_{\nu}(\alpha x)$ may be reduced to the form (21). 


$$
B_{n}=\frac{2 \alpha^{\nu+2} F_{\nu}(\alpha, 1)}{\lambda^{2}\left(\lambda^{2}-\alpha^{2}\right) F_{\nu+1}(\lambda, 1)},
$$

and we know that the series converges to the function in question throughout the interval $0<x<1$. It follows directly from the form of $B_{n}$ and well-known properties of the roots of $J_{\nu}(\lambda)$ that the series converges uniformly throughout the interval $0 \leqq x \leqq 1$. Hence it represents $J_{\nu}(\alpha x)$ $-x^{\nu} J_{\nu}(\alpha)$ in that interval, and our lemma is proved.

Lemma 4. If in the interval $0 \leqq x \leqq 1, \psi(x)$ is defined and such that $x \psi(x)$ has a Lebesgue integral in this interval, and if furthermore

$$
\int_{0}^{1} x \psi(x) F_{v}\left(\lambda_{n}, x\right) d x=0 \quad(n=1,2,3, \cdots),
$$

where $\lambda_{1}, \lambda_{2}, \lambda_{3}, \cdots$ are the successive roots, positive or zero, of equation (3), then $\psi(x)$ is zero at every point of the interval $0<x<1$ at which it is continuous; it is zero for $x=1$ if continuous for that value and the $l$ of equation $(3) \neq 0$, or if it is continuous at that point and at an infinite number of points in its neighborhood; it is zero for $x=0$ if it is continuous at that point and at an infinite number of points in its neighborhood.

The proof is somewhat different in the two cases where the $l$ of equation (3) is different from or is equal to zero. We shall consider first the former case.

By Lemma 2 the function $J_{\nu}(\alpha x)$ may be expanded into a series of the form (13) that converges uniformly in the interval $0 \leqq x \leqq 1$. Multiplying the series by $x \psi(x)$ and integrating term by term* from 0 to 1 , we obtain in view of (23)

$$
\int_{0}^{1} x \psi(x) J_{\nu}(\alpha x) d x=0 .
$$

If in this equation we replace $J_{\nu}(\alpha x)$ by its development in a power series, integrate term by term, $\dagger$ and divide through by $\alpha^{\nu}$, we obtain

$$
0=\sum_{i=1}^{\infty} \frac{(-1)^{i} \alpha^{2 i}}{2^{\nu+2 i} i ! \Gamma(\nu+i+1)} \int_{0}^{1} x^{\nu+1+2 i} \psi(x) d x,
$$

* It follows from the uniform convergence of the series for $J_{\nu}(\alpha x)$ and the fact that $x \psi(x)$ has a Lebesgue integral in the interval $0 \leqq x \leqq 1$ that we may obtain the value of the integral on the left hand side of (24) by integrating term by term.

$t$ The justification is the same as before. 
and this equation is known to hold for all real values of $\alpha$ except zero. Since both sides of the equation are analytic functions, it follows that the equation holds for all values of $\alpha$ and therefore that the right-hand side is identically zero. Hence we have

$$
\int_{0}^{1} x^{\nu+1+2 i} \psi(x) d x=0 \quad(i=1,2,3, \cdots),
$$

and therefore $\psi(x)$ is zero at every point of the interval $0<x \leqq 1$ at which it is continuous, ${ }^{*}$ and is zero for $x=0$ if continuous at that point and at an infinite number of points in its neighborhood.

Let us now consider the case where the $l$ of equation (3) is zero. Making use of Lemma 3 in the same way as we made use of Lemma 2 in the discussion of the previous case, we obtain in place of equation (25) the relation

$$
\int_{0}^{1} x^{\nu+1}\left(x^{2 i}-1\right) \psi(x) d x=0 \quad(i=1,2,3, \cdots) .
$$

From this we readily obtain

$$
\int_{0}^{1} x^{\nu+1+2 i}\left(x^{2}-1\right) \psi(x) d x=0 \quad(i=0,1,2, \cdots) .
$$

Hence $\psi(x)$ is zero at every point of the interval $0<x<1$ at which it is continuous, and is zero for $x=0$ or $x=1$ if continuous at these points and at an infinite number of points in their respective neighborhoods. Thus our lemma is completely proved.

We are now ready to prove the following theorem.

Theorem: If the function $f(x)$ defined for the interval $0 \leqq x \leqq 1$ is such that $x f(x)$ has a Lebesgue integral in this interval, and if the series of the form (2) corresponding to $f(x)$ converges or is summable at all points of the interval $0 \leqq x \leqq 1$ at which $f(x)$ is continuous, defines a function $\varphi(x)$ that is continuous at those points $\dagger$ and such that $x \varphi(x)$ has a Lebesgue

* Cf. Lerch, Acta Mathematica, vol. 27 (1903), p. 347. Also the writer, Bulletin, vol. 14 (1908), p. 368 and vol. 15 (1908), p. 116. The proof of the theorem referred to is given in these papers for Riemann integrability; it applies equally well for Lebesgue integrability except at the point $x=0$, where our added condition is necessary to complete the proof in the same way as it is completed in the writer's footnote on p. 371 of the first reference above.

+ The point $x=1$ is to be excepted for the case $l=0$, unless $f(1)=0$. 
integral in the same interval, and can be integrated term by term over this interval after being multiplied through by a function that is continuous there, then the series will converge or be summable to $f(x)$ at all points of the interval $0<x<1$ at which $f(x)$ is continuous; $*$ it will converge or be summable to $f(x)$ for $x=1$ if $f(x)$ is continuous there and $l \neq 0$, or if $f(x)$ is zero for $x=1$, and is continuous at that point and at an infinite number of points in its neighborhood; it will converge or be summable to $f(x)$ for $x=0$ if the function is continuous at that point and at an infinite number of points in its neighborhood.

We have

$$
\varphi(x)=\sum_{n=1}^{\infty} F_{\nu}\left(\lambda_{n}, x\right) \frac{\int_{0}^{1} x f(x) F_{\nu}\left(\lambda_{n}, x\right) d x}{\int_{0}^{1} x\left[F_{\nu}\left(\lambda_{n}, x\right)\right]^{2} d x} .
$$

Multiplying by $x F_{\nu}\left(\lambda_{n}, x\right)$ for $n=1,2,3, \cdots$ and integrating from 0 to 1 , we obtain, in view of the fact that the $F$ 's form an orthogonal set,

$$
\int_{0}^{1} x[\varphi(x)-f(x)] F_{\nu}\left(\lambda_{n}, x\right) d x=0 \quad(n=1,2,3, \cdots) .
$$

From this equation and Lemma 4 our theorem follows at once. University of Cincinnati, June, 1916.

\section{SECOND NOTE ON REMOVABLE SINGULARITIES.}

BY DR. W. E. MILNE.

IN a "Note on removable singularities" $†$ the writer stated a theorem $\ddagger$ concerning removable singularities for functions of several complex variables. An analogous theorem, with less restrictive hypotheses, is the following

* The convergence or summability to $\frac{1}{2}[f(x+0)+f(x-0)]$ at points where $f(x)$ has a finite jump does not follow directly from the present method. It can be obtained from the convergence or summability to $f(x)$ at points of continuity by a method given by the writer on pp. 428-429 of the paper in volume 10 of the Transactions, referred to above.

$\dagger$ Bulletin, vol. 21 (1914), pp. 116-117.

$\$$ The proof there given for this theorem is incomplete, as Dr. Dunham Jackson pointed out. The gap is filled by the proof here given. 